\title{
Commentary \\ Multimorbidity among Latinx-Americans and Asian American/Pacific Islanders
}

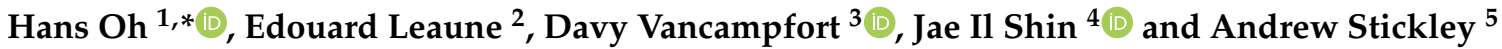 \\ 1 Suzanne Dworak Peck School of Social Work, University of Southern California, Los Angeles, CA 90089, USA \\ 2 PSYR2 Team, Lyon Neuroscience Research Center, F-69000 Lyon, France; edouard.leaune@ch-le-vinatier.fr \\ 3 KU Leuven Department of Rehabilitation Sciences, KU Leuven, Flemish—Brabant, 3000 Leuven, Belgium; \\ davy.vancampfort@kuleuven.be \\ 4 Department of Pediatrics, Yonsei University College of Medicine, Seoul 03722, Korea; shinji@yuhs.ac \\ 5 Stockholm Center for Health and Social Change (SCOHOST), Södertörn University, \\ 14189 Huddinge, Sweden; amstick66@gmail.com \\ * Correspondence: hansoh@usc.edu
}

check for

updates

Citation: Oh, H.; Leaune, E.;

Vancampfort, D.; Shin, J.I.; Stickley, A

Multimorbidity among Latinx-

Americans and Asian American/

Pacific Islanders. J. Ageing Longev.

2022, 2, 26-33. https://doi.org/

$10.3390 /$ jal2010003

Academic Editor:

Francisco Guillén-Grima

Received: 5 October 2021

Accepted: 2 February 2022

Published: 11 February 2022

Publisher's Note: MDPI stays neutral with regard to jurisdictional claims in published maps and institutional affiliations.

Copyright: (C) 2022 by the authors. Licensee MDPI, Basel, Switzerland. This article is an open access article distributed under the terms and conditions of the Creative Commons Attribution (CC BY) license (https:// creativecommons.org/licenses/by/ $4.0 /)$.

\begin{abstract}
Latinx and Asian American/Pacific Islanders (AAPIs) are the fastest growing racial/ethnic populations in the United States, and it is thus increasingly important to address multimorbidity within these populations. However, research has been challenging due to the immigrant health paradox, the variation across ethnic groups, underutilization of treatment, and inadequate mental health assessments. These issues make assessing the prevalence and burden of multimorbidity difficult among Latinx and AAPI populations. Further, racism remains a fundamental cause of health inequity, and should be addressed in policy and practice.
\end{abstract}

Keywords: Latinx Americans; Asian Americans; Pacific Islanders; multimorbidity; health; mental

\section{Introduction}

Multimorbidity is defined as the presence of two or more chronic health conditions [1,2]. Estimates show that multimorbidity has increased in the U.S. over the past two decades, affecting nearly two-thirds of the population [3]. Multimorbidity imposes a considerable economic burden on society [4], and is more difficult to manage than singular conditions, requiring coordinated care across specialists and general practitioners [5]. Practice models are still emerging to more effectively treat people with multimorbidity [6], and failure to effectively manage multimorbidity can be associated with longer hospital stays, lower quality of life, more impairment, and a shorter lifespan [7-10].

While studies show that Black Americans have a higher prevalence of multimorbidity when compared with White Americans, there is some evidence to suggest a slightly lower prevalence among Asian American/Pacific Islanders (AAPIs) [11], with mixed findings for Latinx Americans [3,12]. However, research is generally lacking given the changing demographics of the U.S., especially for the populations that are growing the fastest, which are the Latinx Americans and AAPIs. Between 2000 and 2015, the Latinx American population grew by roughly $60 \%$, while the AAPI population grew by over $70 \%$; it is projected that by 2050, Latinx Americans will constitute nearly a third of the country, and AAPIs will make up almost a tenth [13]. These populations remain under-researched in terms of population health and treatment [14-17], calling for greater clarity around the prevalence and burden of multimorbidity in these groups, bearing in mind several complicating factors. Latinx Americans and AAPIs are similar in many ways, which has naturally led researchers to discuss the groups in tandem (studies are numerous, e.g., [18,19]. Notably, both groups are viewed through the lens of a Black-White racial paradigm [20,21], where Latinx Americans and AAPIs are often situated in a racial dichotomy that undercuts more nuanced views of health disparities and undermines efforts to address health needs. 
In this commentary, we aim to describe four complicating factors that should be taken into consideration when assessing multimorbidity among Latinx Americans and AAPIs, and we highlight the importance of examining racism as a fundamental cause of health inequity.

\subsection{The Health Profiles of Latinx American and AAPI Populations Are Largely Shaped by Immigration}

One commonality between Latinx Americans and AAPIs is that immigration plays a key role in how we understand the health of these populations. Approximately one in three Latinx Americans and almost six in ten AAPIs are foreign-born [22,23]. As such, we cannot discuss the health of these populations without contending with the immigrant health paradox [24]. Immigration is often a stressful process deemed a 'turning point' in the developmental life course [25], resulting in a dramatic upheaval of one's way of life, which according to stress models, should theoretically lead to worse health outcomes when compared with those who do not immigrate. However, studies have shown that immigrants often have a health advantage over their native counterparts [24]. One hypothesis to explain this is that the people who immigrate to the U.S. are often the most capable and healthiest to make the journey, resulting in a selection bias [24]. Another hypothesis suggests that sick and unhealthy immigrants return to their countries of origin, leaving behind only those in good health, which is termed salmon bias [26]. A third hypothesis is that immigrants retain protective aspects of their culture (e.g., diet, customs, cultural proscriptions) that are health-promoting, but eventually lose their health advantage as they assimilate and adopt the health behaviors of the host country [27]. These hypotheses illustrate how complicated it can be to understand the risk and burden of multimorbidity in these populations [24,28], indicating the need for providers to appreciate the complexity of the immigration process [6]: What is the health of Latinx and Asian immigrants upon their arrival? Under what circumstances do they return to their countries of origin? How long have they resided in the United States, and how acculturated are they?

\subsection{There Is Heterogeneity within Racial Groups and across Regions of the U.S.}

Studies on multimorbidity usually fail to make nuanced comparisons across specific ethnic groups to account for significant within-group variation $[17,29]$. Some of this may be a result of practical barriers to studying this variation. While surveys can be administered in Spanish to reach all Hispanic subgroups, it is harder to achieve this among AAPI groups due to language diversity. In other words, surveys must be translated into Chinese, Korean, Vietnamese, Tagalog, Japanese, and so forth, which can be burdensome and expensive. Often, epidemiological surveys provide estimates of multimorbidity that are only generalizable to English-speaking populations.

Setting aside the practical language barriers and sampling challenges, within-group differences in health outcomes are evident [30-33]. For example, some studies suggest that Puerto Ricans have worse health profiles in many respects than other Latinx groups, including higher rates of asthma, Human Immunodeficiency Virus, and psychiatric disorders; meanwhile, Mexican Americans tend to have higher rates of certain conditions, such as diabetes [30,34-36]. Korean Americans drink alcohol at much higher rates than other AAPI groups [37,38], and are at higher risk for certain cancers [39,40]. One study in Northern California found that Pacific Islanders, South Asians, and Filipinos had the highest prevalence and incidence of diabetes among all racial/ethnic groups (even higher than African Americans, Latinx Americans, and Native Americans) [41]. Thus, health profiles of ethnic subgroups are often masked when using monolithic racial categories.

Furthermore, Latinx American and AAPI populations have settled throughout the country according to 'push/pull factors' within historical milieu [42,43]. How ethnic groups fare during the immigration and resettlement process may differ depending on the context of reception, which is the overall valence that the host society directs toward the immigrant group and the opportunity structures available to the immigrants in a given social and geographic location [44]. The context of reception may make one vulnerable to 
insults and hazards, including discrimination and anti-immigrant policies, depending on the neighborhood, city, county, or state, producing different immigrant experiences and ultimately different health profiles.

\subsection{Latinx Americans and AAPIs Underutilize Formal Treatment}

Due to systematic racism [43,45-48], Latinx Americans and AAPI face considerable barriers to treatment $[14,17,43,49-52]$. Studies have shown that AAPIs who needed mental health treatment accessed care at lower rates than whites [53]. Specific AAPI ethnic groups with a high percentage of refugees have additional risk (e.g., Cambodians [54]), and may report receiving low-quality care that does not meet their needs [55]. AAPIs are particularly impacted by cancers, partly attributable to a lack of screening and early intervention [56]. Similarly, Latinx American groups often encounter barriers to accessing high-quality health care services, partly because of low socioeconomic status (i.e., unaffordable out-of-pocket expenses; difficulty navigating complex health care systems), but also due to occupational stratification (e.g., being more likely to work in agriculture, domestic services, food services, construction) where they may be less likely to receive health insurance while facing more occupational hazards. The language barrier has been a problem for both Latinx American and AAPI groups [57-61], and they may also be reluctant to seek services if they are undocumented $[62,63]$.

\subsection{Mental Health among Latinx and AAPI Is Complex}

Measures of multimorbidity vary widely based on lists of conditions that typically include some psychiatric disorders (e.g., depression, schizophrenia). This is problematic in some ways because studies have documented the possibility that Latinx Americans and AAPI may express psychological distress as somatic symptoms [64,65], rendering it harder to make accurate diagnoses. It is possible then, for Latinx Americans and AAPI patients to complain about problems such as indigestion, headaches, and back pain as idioms of distress [66], inadvertently concealing underlying mental health problems. Ultimately, this may result in a singular focus on physical health, and improper or inadequate treatment of mental health. Further, there has been particularly little attention paid to mixed multimorbidity (also known as somatic-mental multimorbidity), which refers to having at least one psychiatric condition and one physical health condition [67], affecting nearly one in five among AAPIs and more than one in four among Latinx Americans.

\section{Measurement Issues}

The two primary ways to measure multimorbidity likely undercount multimorbidity among Latinx Americans and AAPIs due to the aforementioned complicating factors. The first approach to measure multimorbidity is a simple count of diseases, though there is currently little consensus about the exact list of conditions that should be used. Still, simply counting conditions is the most common approach, and is usually based on patient self-report, clinician assessment, or administrative data. This is problematic because, as stated earlier, Latinx Americans and AAPIs underutilize treatment, especially recent immigrants, undocumented immigrants, and those with limited English proficiency. Thus, some Latinx Americans and AAPIs may never receive a formal assessment from a clinician or ever realize they have a diagnosis, and may not accurately assess their own chronic health conditions. Some epidemiological datasets have employed trained lay interviewers to assess for psychiatric disorders [18], but in terms of physical health, still rely on checklists of self-reported physical health conditions (e.g., Has a medical professional ever diagnosed you with diabetes mellitus?). It is possible that analyzing administrative data (e.g., insurance claims, medical records) may provide a more accurate assessment of health conditions when compared with self-reported surveys; however, the data are likely still biased due to treatment gaps $[14,34,49,52,68,69]$. Even among Latinx Americans and AAPI who do seek care, without culturally-responsive providers, we may not know the 
extent to which somatization or culture-bound syndromes (e.g., ataques de nervios) are obfuscating diagnoses.

The second approach to measuring multimorbidity is to develop indices to assess the overall burden of the conditions, weighting certain conditions more heavily based on how they are related to mortality or utilization of resources (i.e., health care costs). The reliability and validity of these measures depend largely on the context (e.g., hospital, primary care, community) and the patient population of interest, which as we have argued is complicated for Latinx Americans and AAPIs given the immigrant paradox, ethnic group variation, treatment gaps, and the tendency for psychosomatic symptoms and culturebound syndromes to obfuscate diagnosis. Weighting certain conditions by accounting for their relations to mortality will likely result in lower index scores for Latinx Americans and AAPI groups; however, it is worth noting that there is some evidence to suggest AAPIs have lower pharmaceutical and physician costs but do not have lower costs associated with hospital stays and emergency department visits [68]. Further, an entirely different picture may emerge if indices accounted for overall impairment, quality of life, and flourishing, or if indices estimated the cost of informal services, such as the burden carried by the family and the larger Latinx American and AAPI communities.

\section{Racism as a Fundamental Cause of Health Inequity}

There has been relatively little research on the risk factors for multimorbidity in general $[2,69]$, and even less on risk factors for multimorbidity among Latinx Americans and Asian Americans, who have unique health profiles and are exposed to different risk factors than their White counterparts. Health inequities persist among Latinx Americans and AAPIs for a host of reasons, including xenophobic policies, barriers to economic and health resources, social stressors (e.g., fear of detention/deportation), economic exploitation, and neighborhood disinvestment, which has shaped risk for multimorbidity across generations $[33,43,45,46]$. Scholars have stressed the importance of addressing multimorbidity by assessing social risk factors [6]. One important determinant of mental and physical health for Latinx Americans and AAPIs is discrimination, which is a relatively common occurrence for Latinx Americans and AAPIs. In the NLAAS data, over 70\% of the Latinx American sample and over three-quarters of the Asian American sample reported some level of discrimination. A large body of literature has identified discrimination as a pernicious social stressor that has been linked to numerous physical and mental health problems [70,71]. Paradies [47] found that when compared with Black Americans, the association between racism and mental health problems was significantly stronger for Latinx Americans and Asian Americans, and the association between racism and physical health was significantly stronger for Latinx Americans.

Prior research has linked perceived discrimination to self-reported mental and physical health [72], as well as singular health conditions, and multimorbidity among Latinx Americans and Asian Americans [73-75]. Gee and colleagues [74] found that self-reported racial discrimination was associated with a two-fold risk for reporting one 12-month psychiatric disorder, and a three-fold risk for reporting two or more psychiatric disorders. In another study, Molina and Simon [75] reported that everyday discrimination was associated with a count of health conditions among Latinx Americans (i.e., physical multimorbidity).

In keeping with the notion that racism is a fundamental cause of health inequalities [48], discrimination is a product of racism and functions as a putative causal factor for multimorbidity through distal and proximal pathways, such as stress processes that wear away at the mind and body over time [25]. However, racism may also impact health by giving rise to unresponsive and discriminatory encounters in health care settings, or by deterring health care utilization in the first place. Ethnic groups may also be differentially exposed to discrimination [76], depending on overall acculturation levels, context of reception, and other ethnocultural characteristics [44]. 


\section{Conclusions}

For Latinx Americans and AAPIs, more research is needed to examine how mental and physical health conditions co-develop, progress, and combine to impact functioning, and how social and environmental factors contribute to the development of multimorbidity. Emerging approaches to population-based multimorbidity care should be contextualized, and may involve expanding home-based care, providing caregiver support, and engaging/coordinating community resources in partnership with Latinx American and AAPI communities.

Author Contributions: Conceptualization, H.O.; Writing—original draft preparation, H.O.; Writingreview and editing, E.L., D.V., J.I.S., A.S. All authors have read and agreed to the published version of the manuscript.

Funding: This research received no external funding.

Institutional Review Board Statement: Not applicable.

Informed Consent Statement: Not applicable.

Data Availability Statement: Not applicable.

Conflicts of Interest: The authors declare no conflict of interest.

\section{References}

1. Goodman, R.A.; Ling, S.M.; Briss, P.A.; Parrish, R.G.; Salive, M.E.; Finke, B.S. Multimorbidity Patterns in the United States: Implications for Research and Clinical Practice; Oxford University Press US: New York, NY, USA, 2015.

2. Salive, M.E. Multimorbidity in Older Adults. Epidemiol. Rev. 2013, 35, 75-83. [CrossRef] [PubMed]

3. King, D.E.; Xiang, J.; Pilkerton, C.S. Multimorbidity Trends in United States Adults, 1988-2014. J. Am. Board Fam. Med. 2018, 31, 503-513. [CrossRef] [PubMed]

4. Picco, L.; Achilla, E.; Abdin, E.; Chong, S.A.; Vaingankar, J.A.; McCrone, P.; Chua, H.C.; Heng, D.; Magadi, H.; Ng, L.L. Economic Burden of Multimorbidity among Older Adults: Impact on Healthcare and Societal Costs. BMC Health Serv. Res. 2016, 16, 173. [CrossRef] [PubMed]

5. Smith, S.M.; Soubhi, H.; Fortin, M.; Hudon, C.; O'Dowd, T. Managing Patients with Multimorbidity: Systematic Review of Interventions in Primary Care and Community Settings. BMJ 2012, 345, e5205. [CrossRef] [PubMed]

6. Savitz, L.A.; Bayliss, E.A. Emerging Models of Care for Individuals with Multiple Chronic Conditions. Health Serv. Res. 2021, 56, 980-989. [CrossRef]

7. Loza, E.; Jover, J.A.; Rodriguez, L.; Carmona, L.; Group, E.S. Multimorbidity: Prevalence, Effect on Quality of Life and Daily Functioning, and Variation of This Effect When One Condition Is a Rheumatic Disease. Semin. Arthritis Rheum. 2009, 38, 312-319. [CrossRef]

8. Menotti, A.; Mulder, I.; Nissinen, A.; Giampaoli, S.; Feskens, E.J.; Kromhout, D. Prevalence of Morbidity and Multimorbidity in Elderly Male Populations and Their Impact on 10-Year All-Cause Mortality: The FINE Study (Finland, Italy, Netherlands, Elderly). J. Clin. Epidemiol. 2001, 54, 680-686. [CrossRef]

9. Nunes, B.P.; Flores, T.R.; Mielke, G.I.; Thume, E.; Facchini, L.A. Multimorbidity and Mortality in Older Adults: A Systematic Review and Meta-Analysis. Arch. Gerontol. Geriatr. 2016, 67, 130-138. [CrossRef]

10. Stickley, A.; Koyanagi, A. Physical Multimorbidity and Loneliness: A Population-Based Study. PLoS ONE 2018, 13, e0191651. [CrossRef]

11. Rocca, W.A.; Boyd, C.M.; Grossardt, B.R.; Bobo, W.V.; Rutten, L.J.F.; Roger, V.L.; Ebbert, J.O.; Therneau, T.M.; Yawn, B.P.; Sauver, J.L.S. Prevalence of Multimorbidity in a Geographically Defined American Population: Patterns by Age, Sex, and Race/Ethnicity. Mayo Clin. Proc. 2014, 89, 1336-1349. [CrossRef]

12. Johnson-Lawrence, V.; Zajacova, A.; Sneed, R. Education, Race/Ethnicity, and Multimorbidity among Adults Aged 30-64 in the National Health Interview Survey. SSM-Popul. Health 2017, 3, 366-372. [CrossRef] [PubMed]

13. Passel, J.S.; D’Vera Cohn, D. US Population Projections, 2005-2050; Pew Research Center: Washington, DC, USA, 2008.

14. Cabassa, L.J.; Zayas, L.H.; Hansen, M.C. Latino Adults' Access to Mental Health Care: A Review of Epidemiological Studies. Adm. Policy Ment. Health Ment. Health Serv. Res. 2006, 33, 316-330. [CrossRef] [PubMed]

15. Chen, M.S. Rectifying Disparities in Funding of Asian American, Native Hawaiian, and Pacific Islander Research by the US National Institutes of Health. JAMA Netw. Open 2019, 2, e197561. [CrossRef] [PubMed]

16. Đoàn, L.N.; Takata, Y.; Sakuma, K.-L.K.; Irvin, V.L. Trends in Clinical Research Including Asian American, Native Hawaiian, and Pacific Islander Participants Funded by the US National Institutes of Health, 1992 to 2018. JAMA Netw. Open 2019, 2, e197432. [CrossRef]

17. Vega, W.A.; Rodriguez, M.A.; Gruskin, E. Health Disparities in the Latino Population. Epidemiol. Rev. 2009, 31, 99-112. [CrossRef] 
18. Alegria, M.; Takeuchi, D.; Canino, G.; Duan, N.; Shrout, P.; Meng, X.-L.; Vega, W.; Zane, N.; Vila, D.; Woo, M.; et al. Considering Context, Place and Culture: The National Latino and Asian American Study. Int. J. Methods Psychiatr. Res. 2004, 13, 208-220. [CrossRef]

19. Kiang, L.; Glatz, T.; Buchanan, C.M. Acculturation Conflict, Cultural Parenting Self-Efficacy, and Perceived Parenting Competence in Asian American and Latino/a Families. Fam. Process 2017, 56, 943-961. [CrossRef]

20. Alcoff, L.M. Latino/as, Asian Americans, and the Black-White Binary. J. Ethics 2003, 7, 5-27. [CrossRef]

21. De Genova, N. Introduction: Latino and Asian Racial Formations at the Frontiers of US Nationalism. In Racial Transformations; Duke University Press: Durham, NC, USA, 2006; pp. 1-20.

22. Noe-Bustamante, L.; Flores, A. Facts on Latinos in the US; Pew Research Center: Washington, DC, USA, 2019.

23. Budiman, A.; Cilluffo, A.; Ruiz, N.G. Key Facts about Asian Origin Groups in the US; Pew Research Center: Washington, DC, USA, 2019.

24. Alcántara, C.; Estevez, C.D.; Alegría, M. Latino and Asian Immigrant Adult Health: Paradoxes and Explanation. In The Oxford Handbook of Acculturation and Health; Schwartz, S.J., Unger, J.B., Eds.; Oxford University Press US: New York, NY, USA, 2017.

25. Elder, G.H., Jr.; George, L.K.; Shanahan, M.J. Psychosocial Stress over the Life Course. In Psychosocial Stress: Perspectives on Structure, Theory, Life-Course, and Methods; Kaplan, H.B., Ed.; Academic Press: Cambridge, MA, USA, 1996.

26. Turra, C.M.; Elo, I.T. The Impact of Salmon Bias on the Hispanic Mortality Advantage: New Evidence from Social Security Data. Popul. Res. Policy Rev. 2008, 27, 515-530. [CrossRef]

27. Abraído-Lanza, A.F.; Chao, M.T.; Flórez, K.R. Do Healthy Behaviors Decline with Greater Acculturation?: Implications for the Latino Mortality Paradox. Soc. Sci. Med. 2005, 61, 1243-1255. [CrossRef]

28. Velasco-Mondragon, E.; Jimenez, A.; Palladino-Davis, A.G.; Davis, D.; Escamilla-Cejudo, J.A. Hispanic Health in the USA: A Scoping Review of the Literature. Public Health Rev. 2016, 37, 31. [CrossRef] [PubMed]

29. Kim, G.; Chiriboga, D.A.; Bryant, A.; Huang, C.-H.; Crowther, M.; Ma, G.X. Self-Rated Mental Health among Asian American Adults: Association with Psychiatric Disorders. Asian Am. J. Psychol. 2012, 3, 44. [CrossRef]

30. Alegría, M.; Mulvaney-Day, N.; Torres, M.; Polo, A.; Cao, Z.; Canino, G. Prevalence of Psychiatric Disorders across Latino Subgroups in the United States. Am. J. Public Health 2007, 97, 68-75. [CrossRef] [PubMed]

31. Holland, A.T.; Palaniappan, L.P. Problems with the Collection and Interpretation of Asian-American Health Data: Omission, Aggregation, and Extrapolation. Ann. Epidemiol. 2012, 22, 397-405. [CrossRef] [PubMed]

32. Maxwell, A.E.; Crespi, C.M.; Alano, R.E.; Sudan, M.; Bastani, R. Health Risk Behaviors among Five Asian American Subgroups in California: Identifying Intervention Priorities. J. Immigr. Minority Health 2012, 14, 890-894. [CrossRef]

33. Yom, S.; Lor, M. Advancing Health Disparities Research: The Need to Include Asian American Subgroup Populations. J. Racial Ethn. Health Disparities 2021, 1-35. [CrossRef]

34. Alegr, M.; Canino, G.; Vera, M.; Rusch, D.; Ortega, A.N. Mental Health Care for Latinos: Inequalities in Use of Specialty Mental Health Services among Latinos, African Americans, and Non-Latino Whites. Ment. Health Care 2002, 53, 1547-1555. [CrossRef]

35. Carlisle, S.K. Disaggregating Race and Ethnicity in Chronic Health Conditions: Implications for Public Health Social Work. Soc. Work. Public Health 2014, 29, 616-628. [CrossRef]

36. Ortega, A.N.; Feldman, J.M.; Canino, G.; Steinman, K.; Alegría, M. Co-Occurrence of Mental and Physical Illness in US Latinos. Soc. Psychiatry Psychiatr. Epidemiol. 2006, 41, 927-934. [CrossRef]

37. Cook, W.K.; Bond, J.; Karriker-Jaffe, K.J.; Zemore, S. Who's at Risk? Ethnic Drinking Cultures, Foreign Nativity, and Problem Drinking among Asian American Young Adults. J. Stud. Alcohol Drugs 2013, 74, 532-541. [CrossRef]

38. Ruderman, D.E.; Clapp, J.D.; Hofstetter, C.R.; Irvin, V.; Kang, S.; Hovell, M.F. Comparison of Heavy Episodic Drinking Patterns between Korean and Chinese Immigrants. Int. J. Alcohol Drug Res. 2016, 5, 65-71. [CrossRef]

39. Lee, J.; Demissie, K.; Lu, S.-E.; Rhoads, G.G. Cancer Incidence among Korean-American Immigrants in the United States and Native Koreans in South Korea. Cancer Control 2007, 14, 78-85. [CrossRef] [PubMed]

40. Torre, L.A.; Sauer, A.M.G.; Chen, M.S.; Kagawa-Singer, M.; Jemal, A.; Siegel, R.L. Cancer Statistics for Asian Americans, Native Hawaiians, and Pacific Islanders, 2016: Converging Incidence in Males and Females. CA Cancer J. Clin. 2016, 66, 182-202. [CrossRef]

41. Karter, A.J.; Schillinger, D.; Adams, A.S.; Moffet, H.H.; Liu, J.; Adler, N.E.; Kanaya, A.M. Elevated Rates of Diabetes in Pacific Islanders and Asian Subgroups: The Diabetes Study of Northern California (DISTANCE). Diabetes Care 2013, 36, 574-579. [CrossRef] [PubMed]

42. Chiswick, B.R.; Miller, P.W. Where Immigrants Settle in the United States. J. Comp. Policy Anal. Res. Pract. 2004, 6, 185-197. [CrossRef]

43. Gee, G.C.; Ford, C.L. Structural Racism and Health Inequities: Old Issues, New Directions. Du Bois Rev. Soc. Sci. Res. Race 2011, 8, 115-132. [CrossRef]

44. Schwartz, S.J.; Unger, J.B.; Zamboanga, B.L.; Szapocznik, J. Rethinking the Concept of Acculturation: Implications for Theory and Research. Am. Psychol. 2010, 65, 237. [CrossRef]

45. Dennis, A.C.; Chung, E.O.; Lodge, E.K.; Martinez, R.A.; Wilbur, R.E. Looking Back to Leap Forward: A Framework for Operationalizing the Structural Racism Construct in Minority and Immigrant Health Research. Ethn. Dis. 2021, 31, 301-310. [CrossRef] 
46. Misra, S.; Kwon, S.C.; Abraído-Lanza, A.F.; Chebli, P.; Trinh-Shevrin, C.; Yi, S.S. Structural Racism and Immigrant Health in the United States. Health Educ. Behav. 2021, 48, 332-341. [CrossRef]

47. Paradies, Y. A Systematic Review of Empirical Research on Self-Reported Racism and Health. Int. J. Epidemiol. 2006, 35, 888-901. [CrossRef]

48. Phelan, J.C.; Link, B.G. Is Racism a Fundamental Cause of Inequalities in Health? Annu. Rev. Sociol. 2015, 41, 311-330. [CrossRef]

49. Matsuoka, J.K.; Breaux, C.; Ryujin, D.H. National Utilization of Mental Health Services by Asian Americans/Pacific Islanders. J. Community Psychol. 1997, 25, 141-145. [CrossRef]

50. Trinh-Shevrin, C.; Islam, N.S.; Rey, M.J. Asian American Communities and Health: Context, Research, Policy, and Action; John Wiley \& Sons: Hoboken, NJ, USA, 2009; Volume 22.

51. Vega, W.A.; Amaro, H. Latino Outlook: Good Health, Uncertain Prognosis. Annu. Rev. Public Health 1994, 15, 39-67. [CrossRef] [PubMed]

52. Wang, P.S.; Berglund, P.; Olfson, M.; Pincus, H.A.; Wells, K.B.; Kessler, R.C. Failure and Delay in Initial Treatment Contact after First Onset of Mental Disorders in the National Comorbidity Survey Replication. Arch. Gen. Psychiatry 2005, 62, 603-613. [CrossRef]

53. Yang, K.G.; Rodgers, C.R.; Lee, E.; Lê Cook, B. Disparities in Mental Health Care Utilization and Perceived Need among Asian Americans: 2012-2016. Psychiatr. Serv. 2020, 71, 21-27. [CrossRef]

54. Sharif, M.Z.; Biegler, K.; Mollica, R.; Sim, S.E.; Nicholas, E.; Chandler, M.; Ngo-Metzger, Q.; Paigne, K.; Paigne, S.; Sorkin, D.H. A Health Profile and Overview of Healthcare Experiences of Cambodian American Refugees and Immigrants Residing in Southern California. J. Immigr. Minority Health 2019, 21, 346-355. [CrossRef]

55. Ngo-Metzger, Q.; Legedza, A.T.; Phillips, R.S. Asian Americans' Reports of Their Health Care Experiences. J. Gen. Intern. Med. 2004, 19, 111-119. [CrossRef]

56. Chen, M.S., Jr. Cancer Health Disparities among Asian Americans: What We Know and What We Need to Do. Cancer 2005, 104, 2895-2902. [CrossRef]

57. Bleakley, H.; Chin, A. Age at Arrival, English Proficiency, and Social Assimilation among US Immigrants. Am. Econ. J. Appl. Econ. 2010, 2, 165-192. [CrossRef]

58. Fiscella, K.; Franks, P.; Doescher, M.P.; Saver, B.G. Disparities in Health Care by Race, Ethnicity, and Language among the Insured: Findings from a National Sample. Med. Care 2002, 40, 52-59. [CrossRef]

59. Kim, G.; Loi, C.X.A.; Chiriboga, D.A.; Jang, Y.; Parmelee, P.; Allen, R.S. Limited English Proficiency as a Barrier to Mental Health Service Use: A Study of Latino and Asian Immigrants with Psychiatric Disorders. J. Psychiatr. Res. 2011, 45, 104-110. [CrossRef] [PubMed]

60. Krogstad, J.M.; Stepler, R.; Lopez, M.H. English Proficiency on the Rise among Latinos; Pew Research Center: Washington, DC, USA, 2015.

61. Sentell, T.; Shumway, M.; Snowden, L. Access to Mental Health Treatment by English Language Proficiency and Race/Ethnicity. J. Gen. Intern. Med. 2007, 22, 289-293. [CrossRef] [PubMed]

62. Kullgren, J.T. Restrictions on Undocumented Immigrants' Access to Health Services: The Public Health Implications of Welfare Reform. Am. J. Public Health 2003, 93, 1630-1633. [CrossRef] [PubMed]

63. Monga, P.; Keller, A.; Venters, H. Prevention and Punishment: Barriers to Accessing Health Services for Undocumented Immigrants in the United States. Laws 2014, 3, 50-60. [CrossRef]

64. Bagayogo, I.P.; Interian, A.; Escobar, J.I. Transcultural Aspects of Somatic Symptoms in the Context of Depressive Disorders. Cult. Psychiatry 2013, 33, 64-74.

65. Lin, E.H.; Carter, W.B.; Kleinman, A.M. An Exploration of Somatization among Asian Refugees and Immigrants in Primary Care. Am. J. Public Health 1985, 75, 1080-1084. [CrossRef]

66. Parsons, C.D.; Wakeley, P. Idioms of Distress: Somatic Responses to Distress in Everyday Life. Cult. Med. Psychiatry 1991, 15, 111-132. [CrossRef]

67. Bobo, W.V.; Yawn, B.P.; St. Sauver, J.L.; Grossardt, B.R.; Boyd, C.M.; Rocca, W.A. Prevalence of Combined Somatic and Mental Health Multimorbidity: Patterns by Age, Sex, and Race/Ethnicity. J. Gerontol. Ser. A Biomed. Sci. Med. Sci. 2016, 71, $1483-1491$. [CrossRef]

68. Chen, J.; Vargas-Bustamante, A.; Ortega, A.N. Health Care Expenditures among Asian American Subgroups. Med. Care Res. Rev. 2013, 70, 310-329. [CrossRef]

69. Marengoni, A.; Angleman, S.; Melis, R.; Mangialasche, F.; Karp, A.; Garmen, A.; Meinow, B.; Fratiglioni, L. Aging with Multimorbidity: A Systematic Review of the Literature. Ageing Res. Rev. 2011, 10, 430-439. [CrossRef]

70. de Freitas, D.F.; Fernandes-Jesus, M.; Ferreira, P.D.; Coimbra, S.; Teixeira, P.M.; de Moura, A.; Gato, J.; Marques, S.C.; Fontaine, A.M. Psychological Correlates of Perceived Ethnic Discrimination in Europe: A Meta-Analysis. Psychol. Violence 2018, 8, 712. [CrossRef]

71. Pascoe, E.A.; Smart Richman, L. Perceived Discrimination and Health: A Meta-Analytic Review. Psychol. Bull. 2009, 135, 531. [CrossRef] [PubMed]

72. Siordia, C.; Covington-Ward, Y.D. Association between Perceived Ethnic Discrimination and Health: Evidence from the National Latino and Asian American Study (NLAAS). J. Frailty Aging 2016, 5, 111-117. [CrossRef] [PubMed] 
73. Gee, G.C.; Spencer, M.S.; Chen, J.; Takeuchi, D. A Nationwide Study of Discrimination and Chronic Health Conditions among Asian Americans. Am. J. Public Health 2007, 97, 1275-1282. [CrossRef] [PubMed]

74. Gee, G.C.; Spencer, M.; Chen, J.; Yip, T.; Takeuchi, D.T. The Association between Self-Reported Racial Discrimination and 12-Month DSM-IV Mental Disorders among Asian Americans Nationwide. Soc. Sci. Med. 2007, 64, 1984-1996. [CrossRef] [PubMed]

75. Molina, K.M.; Simon, Y. Everyday Discrimination and Chronic Health Conditions among Latinos: The Moderating Role of Socioeconomic Position. J. Behav. Med. 2014, 37, 868-880. [CrossRef]

76. Pérez, D.J.; Fortuna, L.; Alegria, M. Prevalence and Correlates of Everyday Discrimination among US Latinos. J. Community Psychol. 2008, 36, 421-433. [CrossRef] 